\title{
Faunenverarmung in einem Schlickgebiet in der Nähe Helgolands
}

\author{
E. RACHOR \\ Institut für Meeresforschung Bremerhaven; \\ Bremerbaven, Bundesrepublik Deutschland
}

\begin{abstract}
Faunal impoverishment in a mud area near Helgoland. Variations in abundance and species composition of macro-zoobenthos were studied at a sublittoral muddy-bottom station in the inner part of the German Bight from 1969 to 1976 . The fauna of this area can be included in the classical Abra alba community. A total of 98 species were found in 62 samples. Of these, only 4 species occurred in all samples: Nucula nitidosa, Diastylis ratbkei, Opbiura texturata and Nephtys hombergii. Species richness has declined since 1969. Populations of several species disappeared in the investigated area and its surroundings. Other populations are shown to exhibit remarkable fluctuations in abundance with sometimes heavy spatfalls or sudden breakdowns in population density, mainly during late summer. The long-term trend of impoverishment of the fauna is attributed to the development of anaerobic conditions in the muddy sediment. Such conditions are favoured by stable, thermohaline stratifications of the water during summer, resulting in low oxygen levels and, probably, enrichment of hydrogen sulfide even in near-bottom water. Moreover, both continuous influx into the area of Elbe water, rich in suspended matter and nutrients and dumping of sewage sludge about $8 \mathrm{~km}$ east of the investigated area are considered to have increased the stress on the bottom fauna.
\end{abstract}

\section{EINLEITUNG}

Allein langfristig konzipierte, kontinuierliche Bestandserhebungen erlauben sichere Aussagen über längerdauernde Veränderungen in den Assoziationsstrukturen des marinen Benthos sowie über Trends in der Entwicklung von Populationen. Sie befreien von Zufälligkeiten, mit denen bei kurzfristig konzipierten Forschungen grundsätzlich zu rechnen ist.

In der Deutschen Bucht hat eine 25jährige Probenserie (1950-1974) von Ziegelmeier bisher sehr aufschlußreiche Ergebnisse über die Beeinträchtigung des Makrobenthos im Sublitoral vor der nordfriesischen Küste durch extrem kalte Winterperioden erbracht und zudem neue Einblicke in die Fragen der Wiederbesiedlung und der gegenseitigen Beeinflussung verschiedener Bodentiere gewährt (Ziegelmeier, 1963, 1964, 1970). Vom Institut für Meeresforschung in Bremerhaven werden die Bodentierbestände des Sublitorals der Deutschen Bucht seit 1966 untersucht, wobei zunächst von Stripp (1969a-c) eine detaillierte Beschreibung der Assoziationen im inneren Teil der Bucht erstellt wurde. Seit 1969 werden von uns an einigen Dauerstationen regelmäßig 
Makrobenthos-Proben gesammelt, die u. a. Ergebnisse über die Beeinträchtigung von Benthospopulationen durch sturmbedingte Bodenerosion (Rachor \& Gerlach, im Druck), über Produktionsleistungen einzelner Populationen (Klein et al., 1975; Rachor, 1976), über die Variationen von Muschelpopulationen (Rachor \& Salzwedel, 1976) und über die Auswirkungen von Abwässern aus der Titandioxidproduktion (Rachor \& Dethlefsen, 1974) erbrachten.

In einer Studie über Populationsdynamik und Produktivität der Muschel Nucula nitidosa Winckworth, 1930, (syn. N. nitida Sowerby, 1833, syn. N. turgida Leckenby \& Marshall, 1875) aus dem Schlickgebiet südöstlich von Helgoland wurde darauf hingewiesen (Rachor, 1976), daß diese Population unter sub-optimalen Bedingungen lebt, was insbesondere im reduzierten Wachstum, im Rückgang der Siedlungsdichte und in einer Abnahme der Produktionsrate zum Ausdruck kommt. Die vorliegende Arbeit beschreibt den allgemeinen Rückgang des Makrobenthos am gleichen Ort und diskutiert diesen Sachverhalt insbesondere im Zusammenhang mit der sommerlichen Belastung des Milieus und den möglichen Auswirkungen von Klärschlammverklappungen in der Nähe des Untersuchungsgebietes.

\section{UNTERSUCHUNGSGEBIET, MATERIAL UND METHODEN}

Die Untersuchungsstation (Abb. 1) liegt in der Schlickregion südöstlich von Helgoland im Zentralgebiet der Abra-alba-Gemeinschaft (sensu Stripp, 1969b). Die Probennahme erfolgte in der Regel mit Bodengreifern nach van Veen, in Einzelfällen mit Kastengreifern nach Reineck (Tab. 1); weitere Einzelheiten über Probennahme und Probenbearbeitung sind in der Arbeit von Rachor (1976) mitgeteilt worden. Daten über die Station sind dort ebenfalls gegeben, so daß hier nur das Wesentliche zusammengefaßt und durch einige zusätzliche Angaben über die Hydrographie ergänzt wird:

$\mathrm{St}$ at i o n s o t : $54^{\circ} 03^{\prime} \mathrm{N}$ und $08^{\circ} 05^{\prime} \mathrm{E}$. Der Ort liegt etwa 8 bis $9 \mathrm{~km}$ westlich vom Verklappungsgebiet für Hamburger Klärschlämme.

Wassertiefe: $23 \mathrm{~m}$. Im Bereich der Station steigt das Bodenprofil schwach von $\mathrm{W}$ nach $\mathrm{E}$ an.

S e d i m e $n t$ : Feinsandiger Schlick (toniger Schluff mit Feinsandbeimengungen, vgl. Gadow, 1968). Dieses feine Sediment ist reich an organischem Detritus und schlecht durchlüftet. In der Regel findet man einen oxydierten Oberflächenbereich von 1 bis $20 \mathrm{~mm}$ Schichtdicke deutlich abgesetzt vom schwärzlich gefärbten, reduzierten Tiefenbereich. Der reduzierte Tiefenbereich fällt durch seinen unangenehmen, $\mathrm{H}_{2} \mathrm{~S}$-dominierten Gestank auf. Gelegentlich wurden Einlagerungen und auch Überschichtungen durch Feinsand festgestellt, insbesondere nach den Wintern 1973/74 und 1975/76. Dieses kann durch Sedimentzufuhr nach sturmbedingten Erosionen im wattennahen Küstenbereich erklärt werden (Reineck, 1963, 1968).

$\mathrm{H}$ y d r og r a p h i : Eine allgemeine, zusammenfassende Beschreibung der Verhältnisse in der Deutschen Bucht ist von Goedecke (1968) gegeben worden. Das hier interessierende Untersuchungsgebiet vor den Flußmündungen von Elbe und Weser hat Lüneburg (1963) beschrieben. Danach liegt das Untersuchungsgebiet im Bereich der Konvergenzzone, des Mischungsgebietes von salzreichem, westlichem und nordwest- 
lichem Nordseewasser und salzarmem, spezifisch leichterem Küstenwasser, das wesentlich von den Zuflüssen aus Elbe und Weser geprägt wird. Wichtig für ökologische Aussagen ist u. a. der Tatbestand, daß es im Bereich der Konvergenzzone regelmäßig zu einer ausgesprochenen thermohalinen Schichtung kommt, wobei in den Monaten April bis Juli maximale thermische Schichtungen auftreten. Die Konvergenzzone ist zudem ein Gebiet sehr hoher Nährstoffanreicherung, Planktonproduktion und auch Planktonzersetzung. Nach Goedecke kann diese Zone wegen der hohen Photosyntheserate auch durch hohe Sauterstoffwerte im Wasser gekennzeichnet werden (Ubersättigung); das O-Überschußgebiet kann bis zum Boden reichen. Goedecke (1968), Lüneburg (1963) und Reineck $(1963,1967)$ stimmen darin überein, daß als eine Erklärung der schlickigen Sedimentbeschaffenheit das Ausfallen von Flußtrübe aus Elbe und Weser im Bereich der Konvergenzzone vor den Flußmündungen anzunehmen ist.

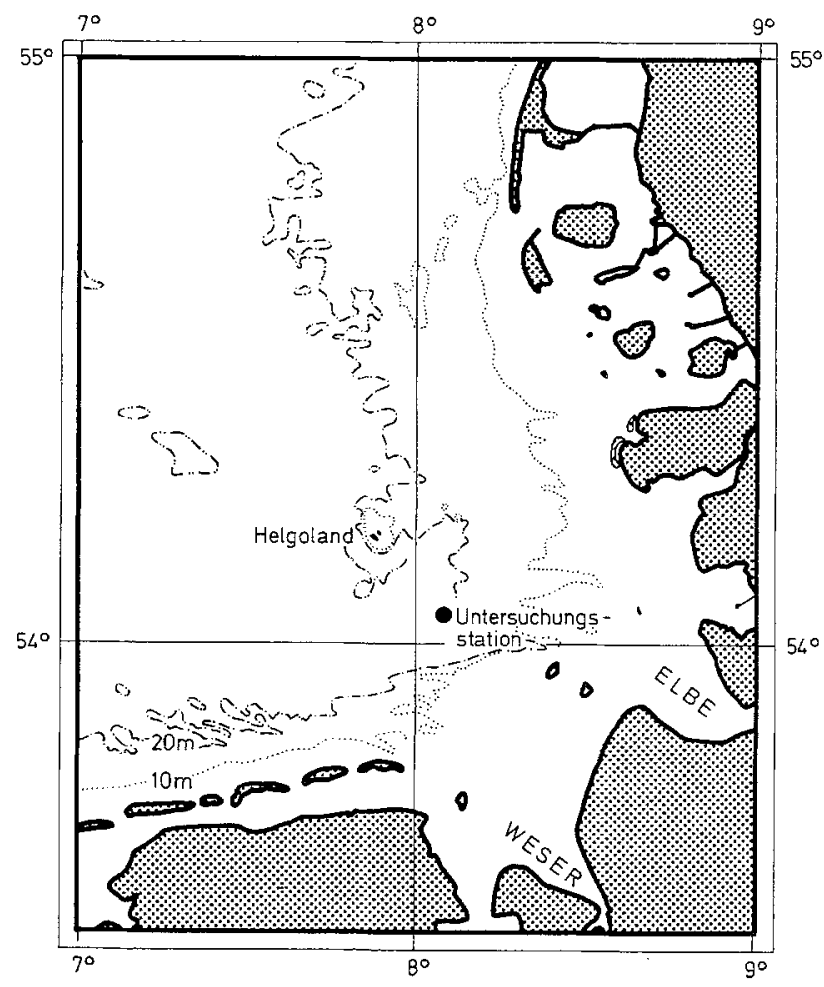

Abb. 1: Lage der Untersuchungsstation in der Deutschen Bucht

\section{ERGEBNISSE}

In den Jahren 1969 bis 1976 wurden an insgesamt 62 Terminen Proben an der Schlickstation genommen; dabei wurden 98 Arten gefunden. 32 Arten sind in mehr als $10 \%$ der Proben vorhanden. Eine Auswahl von Ergebnissen der Aufsammlungen ist in Tabelle 1 zusammengefaßt. 


\section{Tabelle 1}

Sommerliche und spätsommerliche Abundanzwerte (Individuen pro $\mathrm{m}^{2}$ ) der häufigeren $\mathrm{Ma}$ krobenthos-Arten an der Untersuchungsstation im Zeitraum 1969 bis 1976. Die Zahlen von Juli 1966 sind der Arbeit von Stripp (1969b, Tab. 15) entnommen. In den Jahren 1973-1974 wurden nur wenige Proben genommen, so daß die Tabelle hier Lücken aufweist; zum besseren Vergleich ist die Januar-Probe von 1974 aufgenommen worden. Zur Dokumentation der besonders auffälligen Befunde von 1976 sind die Werte für die vier Monate April, Juni, August und September aufgeführt. Es wurde Wert darauf gelegt, nur vergleichbare Proben aufzufüh-

\begin{tabular}{|c|c|c|c|c|c|c|}
\hline Proben-Nr. & & $(71)$ & 19 & 26 & 86 & 98 \\
\hline Datum & $\begin{array}{c}\mathrm{F} \\
(\% / 0)\end{array}$ & $\begin{array}{r}\text { Juli } \\
1966\end{array}$ & $\begin{array}{r}4 \\
\text { Aug. } \\
1969\end{array}$ & $\begin{array}{r}8 \\
\text { Sept. } \\
1969\end{array}$ & $\begin{array}{r}14 \\
\text { Aug. } \\
1970\end{array}$ & $\begin{array}{r}6 \\
\text { Okt. } \\
1970\end{array}$ \\
\hline $\begin{array}{l}\text { Greifer-Typ } \\
\text { Greifer-Anzahl }\end{array}$ & & 0,1 & $\begin{array}{r}0,1 \\
5\end{array}$ & $\begin{array}{r}0,1 \\
5\end{array}$ & $\begin{array}{r}0,1 \\
5\end{array}$ & $\begin{array}{r}0,1 \\
5\end{array}$ \\
\hline Nucula nitidosa Winckworth & 100,0 & 365 & 872 & 694 & 712 & 460 \\
\hline Diastylis ratbkei (Kröyer) & 98,4 & 500 & 58 & 48 & 80 & 36 \\
\hline Opbiura texturata Lamardk & 100,0 & - & 222 & 22 & 26 & 24 \\
\hline Nepbtys hombergii Savigny & 100,0 & 75 & 94 & 110 & 84 & 48 \\
\hline Mysella bidentata Montagu & 79,0 & 15 & 18 & 4 & 14 & 14 \\
\hline Abra alba (Wood) & 77,4 & 10 & 270 & 138 & 78 & 6 \\
\hline Echiurus echiurus (Pallas) & 66,1 & 10 & 354 & 274 & 150 & 68 \\
\hline Harmothoe sarsi sarsi (Kinberg) & 64,5 & - & 104 & 132 & 114 & 56 \\
\hline Pboloe minuta (Fabricius) & 61,3 & - & 2 & 18 & 6 & 18 \\
\hline Phoronis spec. & 56,5 & - & 6 & 2 & - & 54 \\
\hline Opbiura albida Forbes & ca. 54 & 5 & 2 & $3 \overline{6}$ & 2 & - \\
\hline Anaitides groenlandica (Oersted) & 48,4 & 5 & - & - & - & - \\
\hline Gattyana cirrosa (Pallas) & 45,2 & 10 & 46 & 26 & 20 & 2 \\
\hline Abra nitida (O. F. Müller) & 41,9 & 5 & 250 & 214 & - & - \\
\hline Crangon crangon (Linné) & 35,5 & - & 4 & - & - & - \\
\hline Scalibregma inflatum Rathke & 32,3 & 5 & 602 & 436 & 556 & - \\
\hline Polydora ciliata (Johnston) & 22,6 & - & 30 & 50 & 14 & 88 \\
\hline Pectinaria koreni Malmgren & 19,4 & 10 & 16 & 10 & 4 & - \\
\hline Scoloplos armiger (O. F. Müller) & 19,4 & - & 18 & 18 & - & - \\
\hline Nereis virens Sars & 17,7 & - & - & - & - & - \\
\hline Ampelisca brevicornis $\mathrm{Da}$ Costa & 17,7 & - & - & - & 2 & - \\
\hline Ophelina acuminata Oersted & 16,1 & - & 8 & 16 & 一 & - \\
\hline Goniada maculata Oersted & 16,1 & 5 & - & 2 & - & - \\
\hline Eteone longa (Fabricius) & 14,5 & - & - & - & - & - \\
\hline Ampharete acutifrons (Grube) & 14,5 & - & 6 & - & - & - \\
\hline Owenia fusiformis Delle Chiaje & 12,9 & - & 14 & 2 & - & 6 \\
\hline Capitomastus minimus (Langerhans) & 12,9 & - & - & - & 2 & - \\
\hline Lanice conchilega (Pallas) & 11,3 & - & 16 & 6 & - & - \\
\hline Spiophanes bombyx (Claparède) & 11,3 & - & 74 & 66 & - & - \\
\hline Tellina fabula Gmelin & 11,3 & - & 2 & - & - & - \\
\hline Heteromastus flifformis (Claparède) & 11,3 & - & - & - & - & 4 \\
\hline Diastylis bradyi Norman & 11,3 & - & - & - & - & 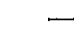 \\
\hline Individuenzahl der selteneren Arten & $(\leq 10)$ & 35 & 128 & 142 & 4 & 14 \\
\hline Gesamt-Individuenzahl & & 1060 & 3216 & 2466 & 1868 & 898 \\
\hline Anzahl der selteneren Arten & & 3 & 8 & 7 & 2 & 5 \\
\hline Gesamt-Artenzahl & & 16 & 32 & 29 & 18 & 19 \\
\hline Reduzierte Artenzahl & & (16) & 27 & 25 & 13 & 14 \\
\hline Diversität ( $\left.\mathrm{H}^{\prime}\right)$ & & 2,04 & 3,40 & 3,45 & 2,54 & 2,63 \\
\hline
\end{tabular}


Tabelle 1

ren (in der Regel 5 van-Veen-Greifer von 0,1 $\mathrm{m}^{2}$ Fläche; lediglich im Juni 1975 sind Werte von kleinen Reineck-Kastengreifern mit $1 / 60 \mathrm{~m}^{2}$ Fläche angegeben). $\mathrm{F}=$ Häufigkeit, bezogen auf die Gesamtheit aller 62 Proben; 0,1 = van-Veen-Greifer von $0,1 \mathrm{~m}^{2}$ Fläche; $\mathrm{KG}=$ kleiner Reineck-Kastengreifer. In den Zahlenwerten von Opbiura texturata sind sämtliche nicht identifazierbaren Jungtiere der Gattung Ophiura enthalten. (Aus Platzgründen kann die Gesamtheit der Daten aller 62 Proben an dieser Stelle nicht wiedergegeben werden; die Zahlen sind in Tabellen zusammengestellt und können vom Autor angefordert werden)

\begin{tabular}{|c|c|c|c|c|c|c|c|c|c|c|c|c|}
\hline 163 & 168 & 252 & 272 & 290 & 297 & 343 & VH-39 & 508 & 334 & 518 & 763 & 807 \\
\hline $\begin{array}{r}29 \\
\text { Juli } \\
1971\end{array}$ & $\begin{array}{r}14 \\
\text { Sept. } \\
1971\end{array}$ & $\begin{array}{r}14 \\
\text { Juni } \\
1972\end{array}$ & $\begin{array}{r}5 \\
\text { Okt. } \\
1972\end{array}$ & $\begin{array}{r}21 \\
\text { Aug. } \\
1973\end{array}$ & $\begin{array}{r}{ }^{4} \\
\text { Jan. }\end{array}$ & $\begin{array}{r}20 \\
\text { Sept. } \\
1974\end{array}$ & $\begin{array}{r}10 \\
\text { Juni } \\
1975\end{array}$ & $\begin{array}{r}24 \\
\text { Nov. } \\
1975\end{array}$ & $\begin{array}{r}23 \\
\text { Apr. } \\
1976\end{array}$ & $\begin{array}{r}22 \\
\text { Juni } \\
1976\end{array}$ & $\begin{array}{r}17 \\
\text { Aug. } \\
1976\end{array}$ & $\begin{array}{r}9 \\
\text { Sept. } \\
1976\end{array}$ \\
\hline $\begin{array}{r}0,1 \\
5\end{array}$ & $\begin{array}{r}0,1 \\
5\end{array}$ & $\begin{array}{r}0,1 \\
5\end{array}$ & $\begin{array}{r}0,1 \\
5\end{array}$ & $\begin{array}{r}0,1 \\
5\end{array}$ & $\begin{array}{r}0,1 \\
6\end{array}$ & $\begin{array}{r}0,1 \\
5\end{array}$ & $\begin{array}{r}K G \\
6\end{array}$ & $\begin{array}{r}0,1 \\
5\end{array}$ & $\begin{array}{r}0,1 \\
5\end{array}$ & $\begin{array}{r}0,1 \\
5\end{array}$ & $\begin{array}{r}0,1 \\
5\end{array}$ & $\begin{array}{r}0,1 \\
8\end{array}$ \\
\hline 106 & 116 & 198 & 356 & 216 & 307 & 134 & 330 & 416 & 2 & 28 & 48 & 165 \\
\hline 80 & 80 & 1398 & 744 & 1214 & 170 & 280 & 770 & 34 & 6 & 420 & 98 & 174 \\
\hline 12 & 32 & 12 & 46 & 88 & 345 & 854 & 230 & 392 & 8 & 8 & 404 & 457 \\
\hline 2 & 24 & 54 & 48 & 50 & 55 & 52 & 160 & 66 & 12 & 12 & 8 & 34 \\
\hline 32 & 42 & 8 & 4 & 6 & 1 & - & - & - & - & - & 34 & 3 \\
\hline 30 & - & 50 & 16 & 6 & - & 46 & 380 & 2 & - & 140 & 114 & 3 \\
\hline 26 & 14 & 82 & 8 & 2 & - & - & - & - & - & 4 & 8 & - \\
\hline 32 & 16 & 50 & 8 & 2 & - & - & - & - & - & 2 & - & - \\
\hline 10 & 12 & - & 2 & - & - & - & - & - & - & 6 & 306 & 2 \\
\hline 30 & 32 & - & - & - & - & - & - & - & - & - & 2 & - \\
\hline- & - & - & 8 & 2 & 9 & 2 & $\ldots$ & - & - & - & 22 & 40 \\
\hline 2 & - & - & - & - & 1 & - & 20 & - & - & 20 & 22 & - \\
\hline- & 2 & - & - & - & - & - & - & - & - & - & - & - \\
\hline 14 & - & - & - & - & 1 & - & - & - & - & 14 & - & 2 \\
\hline 6 & 4 & - & 2 & 16 & 4 & - & - & 4 & - & - & 8 & 3 \\
\hline 50 & - & 174 & - & 2 & - & - & - & - & - & - & - & - \\
\hline - & 8 & - & - & - & - & - & - & - & - & - & - & - \\
\hline- & - & - & - & 一 & - & - & - & - & - & 38 & 2 & - \\
\hline- & - & 2 & - & - & - & - & - & - & - & 4 & - & - \\
\hline 2 & - & - & - & - & - & - & - & - & - & - & - & - \\
\hline- & - & 2 & - & - & 1 & - & - & - & - & - & - & - \\
\hline- & - & 204 & - & - & - & - & - & - & 一 & - & - & - \\
\hline- & - & - & - & - & - & - & - & - & - & - & - & - \\
\hline- & - & - & - & - & - & - & - & - & - & 2 & 4 & - \\
\hline- & - & 6 & - & 8 & - & - & - & - & - & - & - & - \\
\hline 6 & - & - & - & - & - & - & - & 一 & - & - & - & - \\
\hline- & 14 & - & - & - & - & - & - & - & - & - & - & - \\
\hline- & - & - & - & - & - & - & - & - & - & 4088 & 112 & - \\
\hline- & - & - & - & - & - & - & - & - & - & 2 & 12 & - \\
\hline- & - & 2 & - & - & - & - & - & - & - & 112 & 6 & - \\
\hline- & 2 & 一 & - & - & - & - & - & - & - & - & - & - \\
\hline- & - & - & - & - & - & - & - & - & - & - & - & - \\
\hline 2 & 4 & 4 & - & 16 & 1 & - & 10 & 6 & - & 6 & 54 & 6 \\
\hline 442 & 402 & 2246 & 1242 & 1628 & 895 & 1368 & 1900 & 920 & 28 & 4906 & 1264 & 889 \\
\hline 1 & 2 & 2 & - & 6 & 1 & - & 1 & 2 & - & 3 & 11 & 2 \\
\hline 17 & 16 & 16 & 11 & 18 & 11 & 6 & 7 & 8 & 4 & 19 & 28 & 12 \\
\hline 12 & 11 & 11 & 9 & 7 & 5 & 6 & 6 & 4 & 4 & 13 & 21 & 8 \\
\hline 3,34 & 3,15 & 2,02 & 1,59 & 1,38 & 1,92 & 1,58 & 2,21 & 1,60 & 1,79 & 1,08 & 3,39 & 1,96 \\
\hline
\end{tabular}




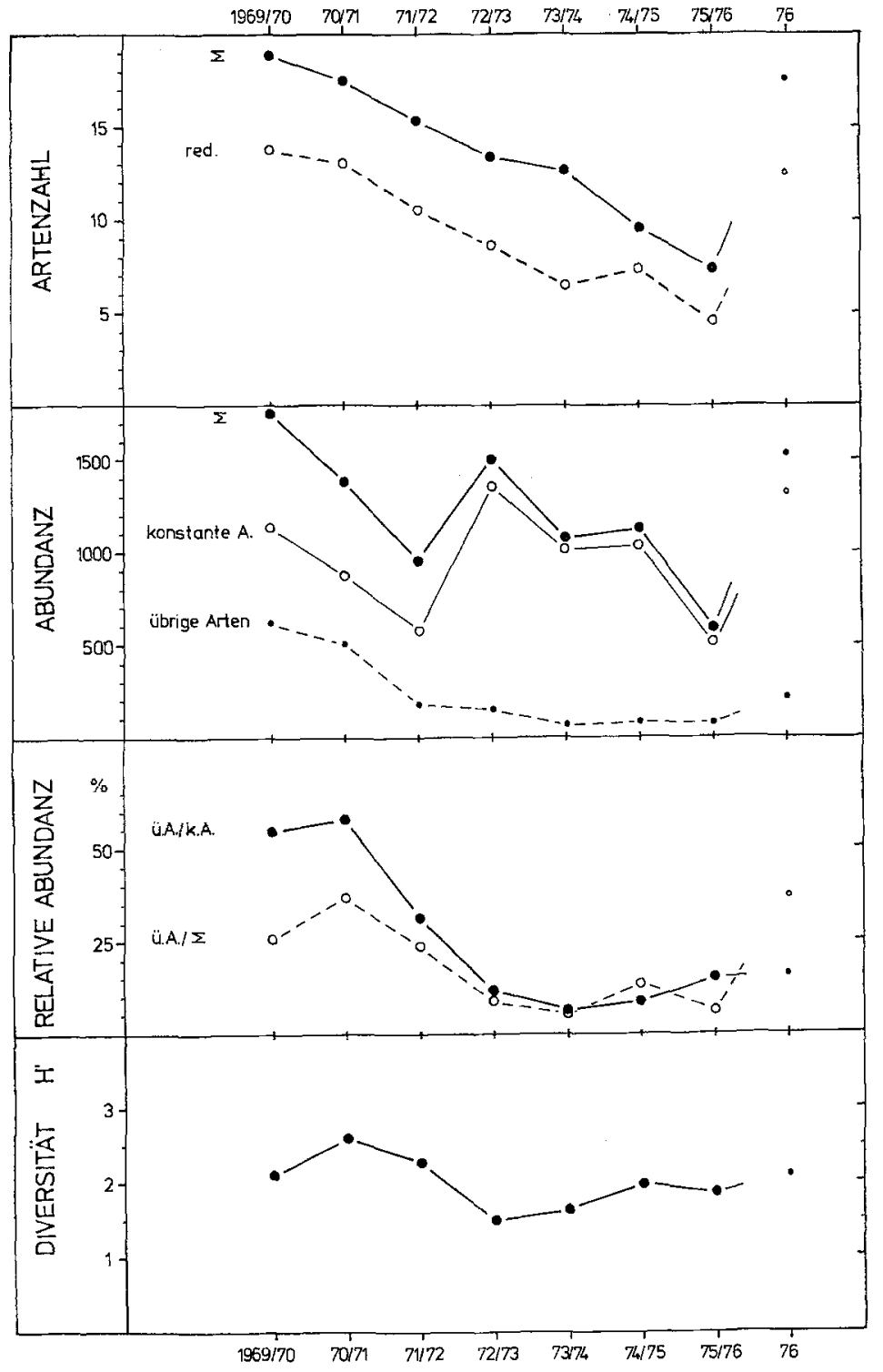

Abb. 2: Jahresmittelwerte der Artenzahl, Abundanz und Diversität an der Untersuchungsstation, Entwidklung von 1969 bis 1976. $\Sigma=$ Gesamtzahl; red. = reduzierte Artenzahl (ohne Einzelfunde und ohne die mit Bodengreifern schwer erfaßbaren Arten); k.A. = konstante Arten (Nucula nitidosa, Diastylis ratbkei, Opbiura texturata und Nepbtys bombergii); ü.A. = übrige Arten. Die Werte sind jeweils für den. Zeitraum Mai bis April gemittelt; für 1976 reichen die Zahlen jedoch nur bis zum September (kleinere Symbole) 


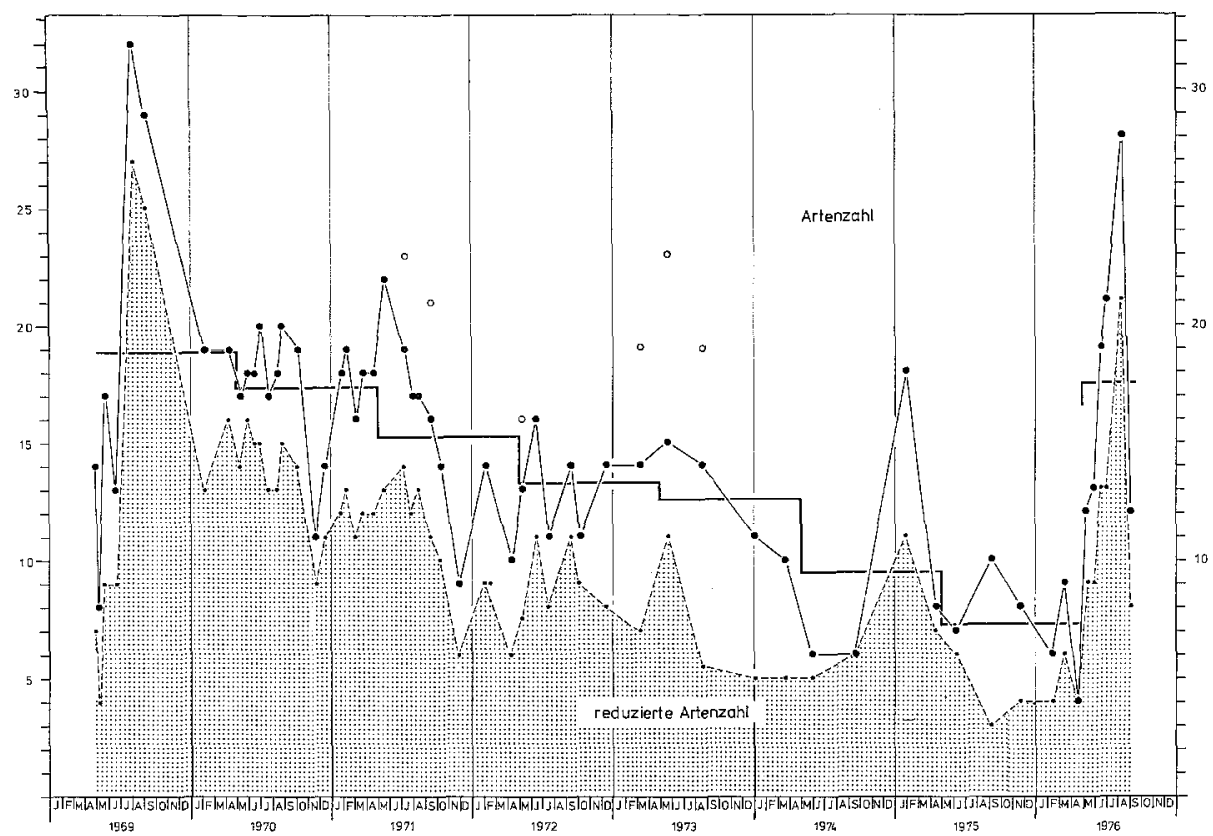

Abb. 3: Artenzahl (Einzelwerte für sämtliche Proben), Entwicklung von 1969 bis 1976. Offene Kreise $=$ Werte aus sehr umfangreichen Proben (wenigstens 10 Greifer); stufenförmige Kurve $=$ Jahresmittelwerte

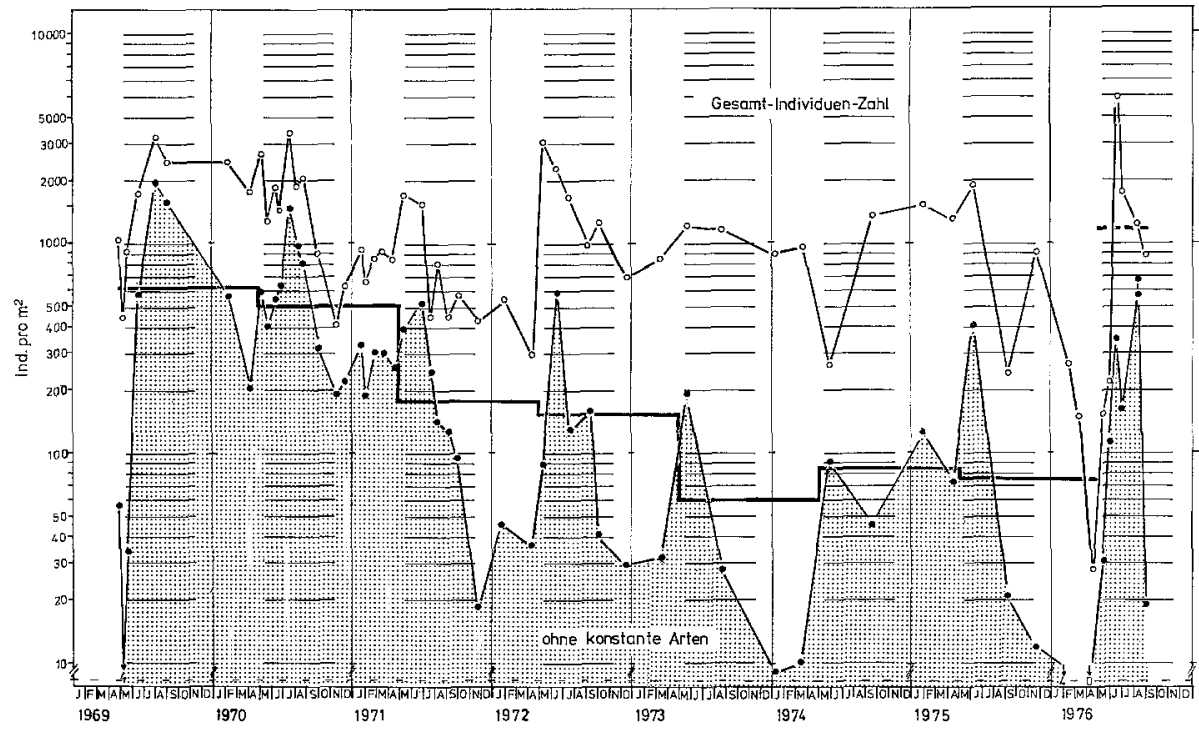

Abb. 4: Gesamtabundanz und Abundanz der nicht konstanten Arten (Einzelwerte für sämtliche Proben), Entwicklung von 1969 bis 1976. Stufenförmige Kurve $=$ Jahresmittelwerte ohne konstante Arten 
Vier Arten sind mit großer Konstanz gefunden worden (in 98,4 und 100\% aller Proben): Nucula nitidosa, Diastylis ratbkei, Ophiura texturata und Nephtys hombergii. Sie dominieren nach Zahl und Gewicht und machen im Schnitt aller Proben $77 \%$ aller Individuen aus.

Der Zahlenanteil der weniger konstanten Arten schwankt zwischen 0 (April 1976) und $93 \%$ (Juli 1976); das Jahresmittel dieses Zahlenanteils zeigt jedoch insgesamt eine fallende Tendenz mit einem geringen Anstieg in der Periode Mai 1974 bis April 1975 und einem starken Anstieg im frühen Sommer 1976 (Abb. 2). Etwa gleichsinnig verlaufen die Änderungen in der Diversität $\left(\mathrm{H}^{\prime}=\right.$ Species-Diversität nach der ShannonWiener-Funktion, Pielou, 1969); allerdings ist 1976 in der Diversität der Anstieg auf Grund der Massenentwicklung von Lanice conchilega weniger ausgeprägt.

Dieser Trend zur Verarmung der Makrofauna wird am deutlichsten durch die Darstellung der Artenzahl wiedergegeben. Betrachtet man nur die Jahresmittel (Mai bis April), so sinkt die Artenzahl von fast 19 in der Periode 1969/70 auf nahezu 7 in 1975/76. Auch wenn man alle Einzelfunde von Arten in einer Probe wegläßt und darüber hinaus solche Tiere, die mit Bodengreifern nicht sicher erfaßbar sind (z. B. Crustacea wie Crangon und Mysidacea), zeigt diese "reduzierte Artenzahl" den gleichen starken Trend. Hier ist zu erwähnen, daß im Jahre 1966 mit nur zwei Bodengreifern an der gleichen Stelle 16 Arten registriert wurden (Stripp, 1969b), 1967 nördlich davon 18 Arten (Dörjes, 1968). Bei der Betrachtung der Einzelwerte fallen die durch Jahreszeiten und auch Probenumfang bedingten Schwankungen in der Artenzahl sehr auf. Als charakteristisch kann ein Jahresgang angesehen werden, in dem im frühen Sommer die Artenzahl ansteigt (insbesondere 1969, 1970, 1973, 1975 und 1976) und dann auch noch im Sommer (August-September) wieder stark abnimmt (sehr deutlich 1971). Auch nach der starken Zunahme der Artenzahl im Sommer 1976 ist schon im September wieder ein niedriger Wert in der Nähe des Mittelwertes des vorhergehenden Jahres erreicht worden (Abb. 3).

Auch in der Gesamt-Abundanz ist insgesamt ein Rückgang zu verzeichnen, und zwar zunächst stark auf Kosten der weniger konstanten Arten, ab 1973 aber auch bei den konstanten Arten (Abb. 2 und 4).

Die Abundanzentwicklung der konstanten Arten ist im Detail in Abbildung 5-8 dargestellt. Für die langlebige, dominierende Muschel Nucula nitidosa ist ein langsamer Rückgang feststellbar, der nur durch die immer wieder erfolgenden Brutschübe überlagert ist - die Art ist im Untersuchungsgebiet vor allem Herbst- und Winterlaicher (Rachor, 1976). Im April 1976 wurde ein absolutes Abundanzminimum mit 2 Individuen pro $\mathrm{m}^{2}$ erreicht; danach $\mathrm{kam}$ es durch die nachgewachsene Brut $\mathrm{zu}$ einer gewissen Erholung. Die Jahres-Produktion ging von mehr als $18 \mathrm{kcal}$ pro $\mathrm{m}^{2}$ in der Periode 1970/71 auf weniger als $3 \mathrm{kcal}$ in der Periode 1973/74 zurïck (Rachor, 1976).

Die Siedlungsdichte des Schlangensternes Opbiura texturata nahm von 1969 bis zum Frühjahr 1973 stark ab; dann kam es zu einer kräftigen Erholung, die nur im Frühling und Frühsommer 1976 durch einen auffälligen Rückgang unterbrochen ist.

Die Cumacee Diastylis ratbkei ist durch deutliche jahreszeitliche Zyklen in der Abundanz gekennzeichnet; die Zahlen steigen im Mai-Juni nach Schlüpfen der Brut stark an. Ein durchgehender, abnehmender Trend ist nicht feststellbar, eher eine gewisse Zunahme ab Sommer 1972. 


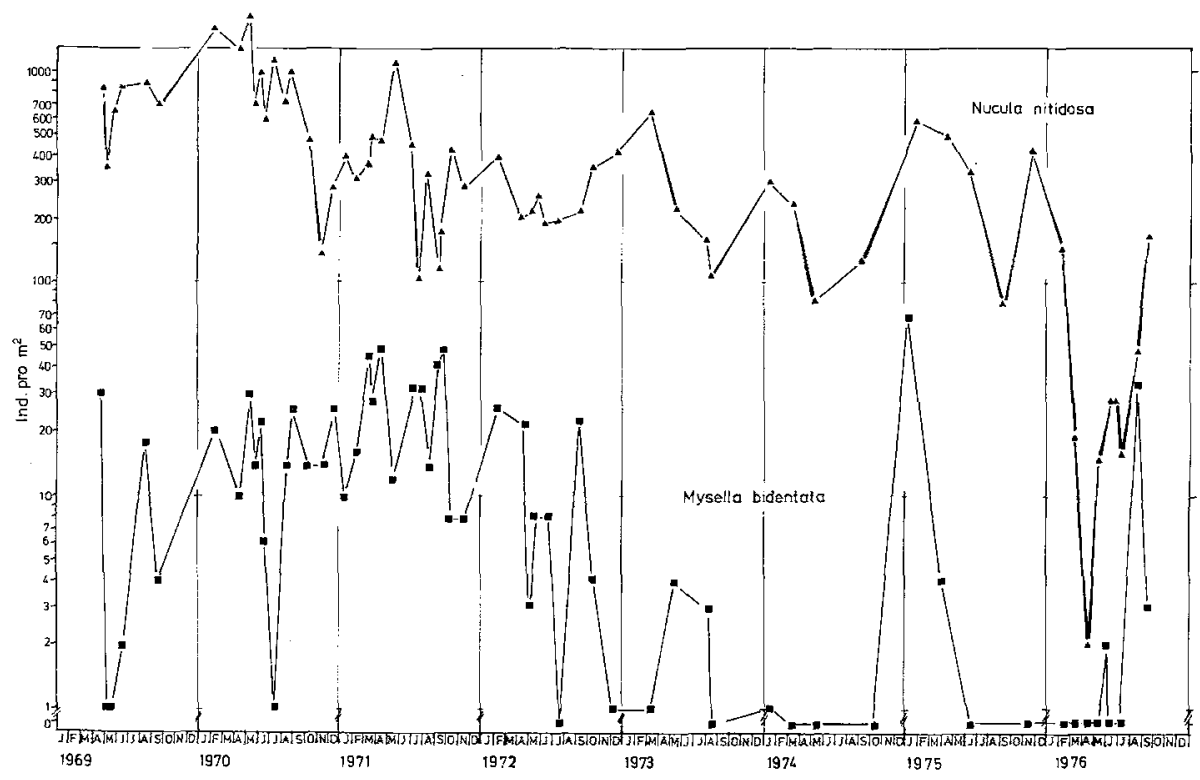

Abb. 5: Abundanz von Nucula nitidosa und Mysella bidentata (Bivalvia), Entwicklung von 1969 bis 1976

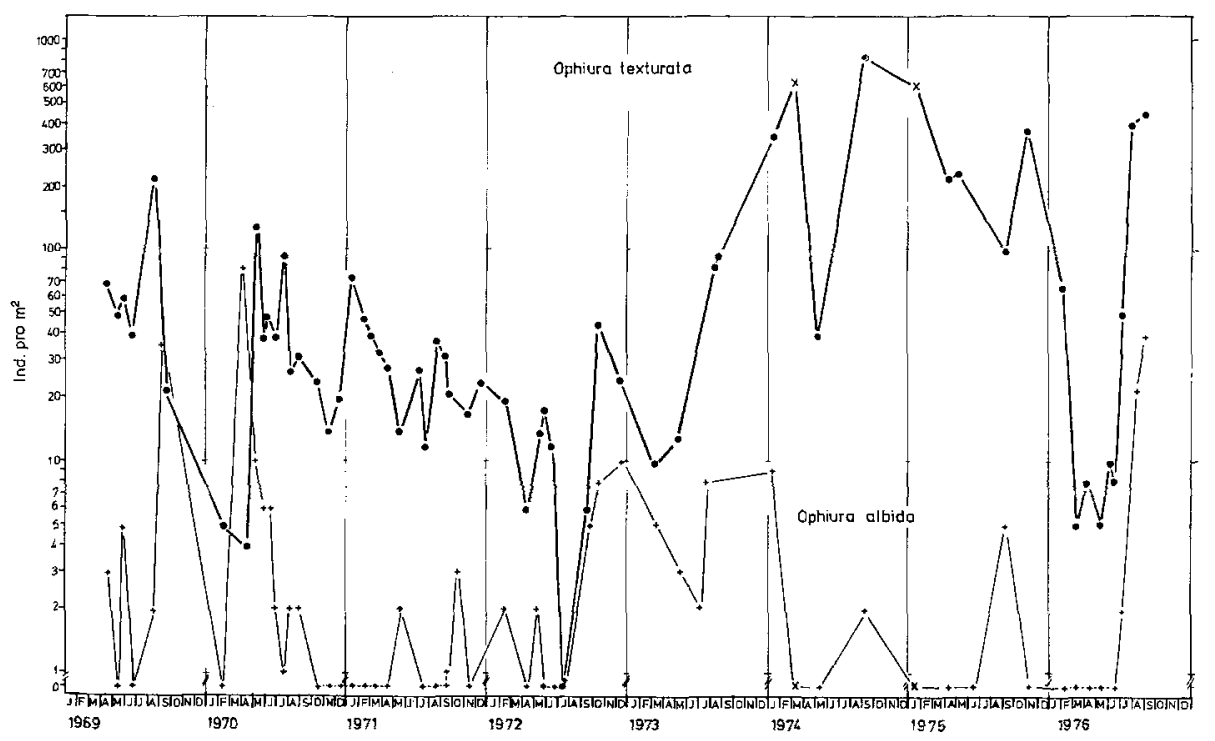

Abb. 6: Abundanz von Opbiura texturata und O. albida (Echinodermata), Entwicklung von 1969 bis 1976 


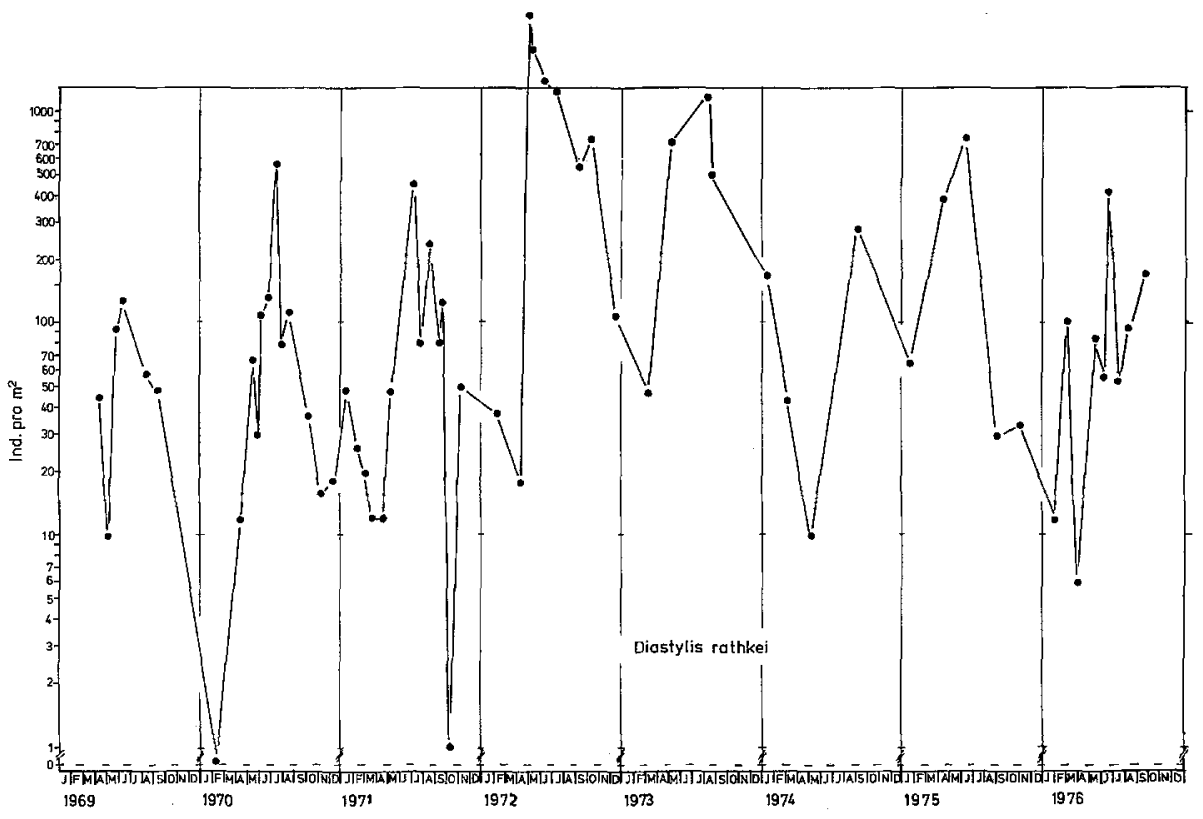

Abb. 7: Abundanz von Diastylis ratbkei (Crustacea), Entwicklung von 1969 bis 1976

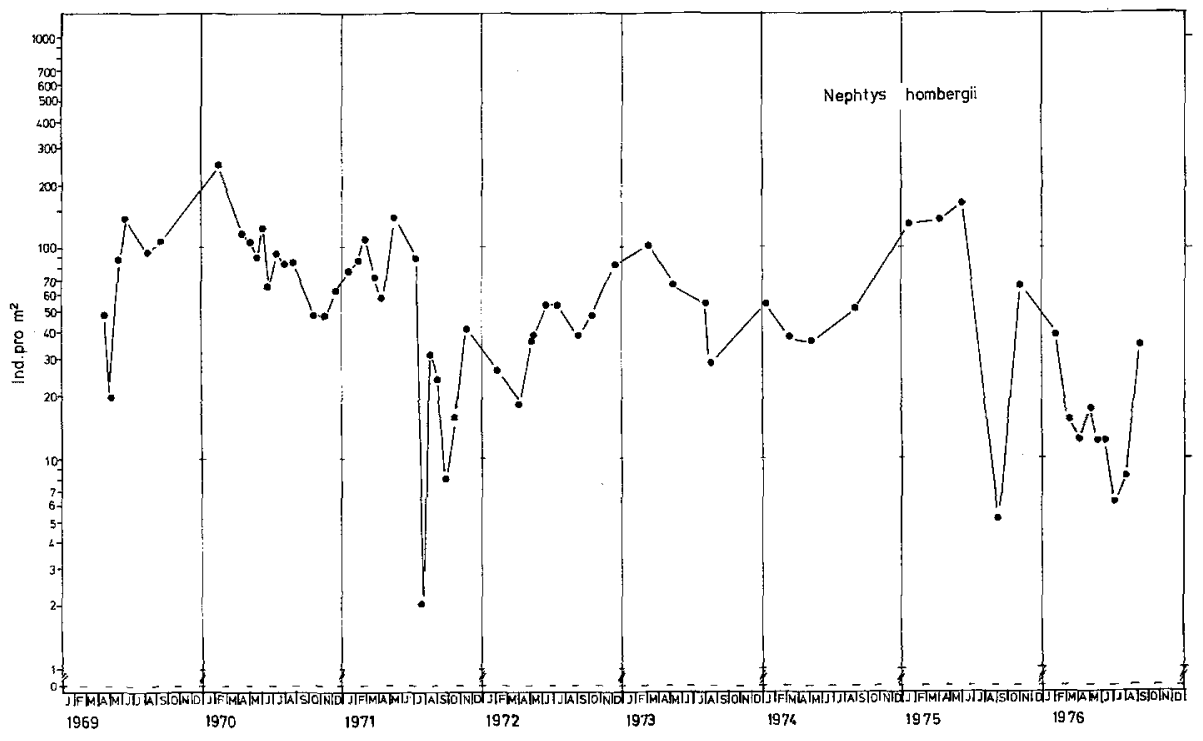

Abb. 8: Abundanz von Nephtys bombergii (Polychaeta), Entwicklung von 1969 bis 1976 
Der Polychaet Nephtys bombergii zeigt keine deutlichen Langzeittrends. Die Dichtemaxima im Jahresgang liegen in den Wintermonaten und im Frühjahr. In den Sommern 1971, 1975 und 1976 sind starke Einbrüche in der Siedlungsdichte zu verzeichnen. Offensichtlich kommt es danach noch zu einer Neubesiedlung durch Jungtiere.

Bei den Arten geringer Konstanz ist das Bild nicht einheitlich, jedoch können Gruppen mit gleichartiger Abundanzentwicklung aufgezeigt werden:

(a) Arten mit anfangs gut entwickelten, konstanten Beständen, die im Spätsommer 1971 zusammenbrechen, sich in den beiden anschließenden Jahren trotz Wiederbesiedlung nicht erholen können und 1974 und 1975 fehlen. Im Frühsommer 1976 beginnt eine erneute Wiederbesiedelungsphase, doch brechen die neuen Bestände offensichtlich schon im Hochsommer wieder zusammen: Echiurus echiurus, Harmothoe sarsi, Gattyana cirrosa, Pholoe minuta (Abb. 9-12).

(b) Arten, deren Populationen erst nach 1972 stark in der Abundanz zurückgehen: Mysella bidentata, Phoronis spec. (vgl. Abb. 5 unten).

(c) Abra alba als stark fluktuierende Art mit gelegentlichen Massenentwicklungen und wiederholten Zusammenbrüchen der Population im Hochsommer (Abb. 13).

(d) Fluktuierende Arten mit mehr oder weniger hohen Besiedlungsdichten in den Sommern der ersten Jahre, die aber später fehlen: Scalibregma inflatum, Polydora ciliata $(=P$. ligni $)$, Pectinaria koreni und mit Einschränkungen auch Scoloplos armiger als weiterer Polychaet sowie die Muschel Abra nitida. Es fällt auf, daß auch bei diesen Arten die Populationen vor allem im Hochsommer schwinden.

(e) Arten ohne Regelmäßigkeit in der Abundanzdynamik, jedoch mit zuweilen starken bis Massen-Entwicklungen im Frühsommer: Opbelina acuminata $(1969,1972)$ und Lanice conchilega (1976).

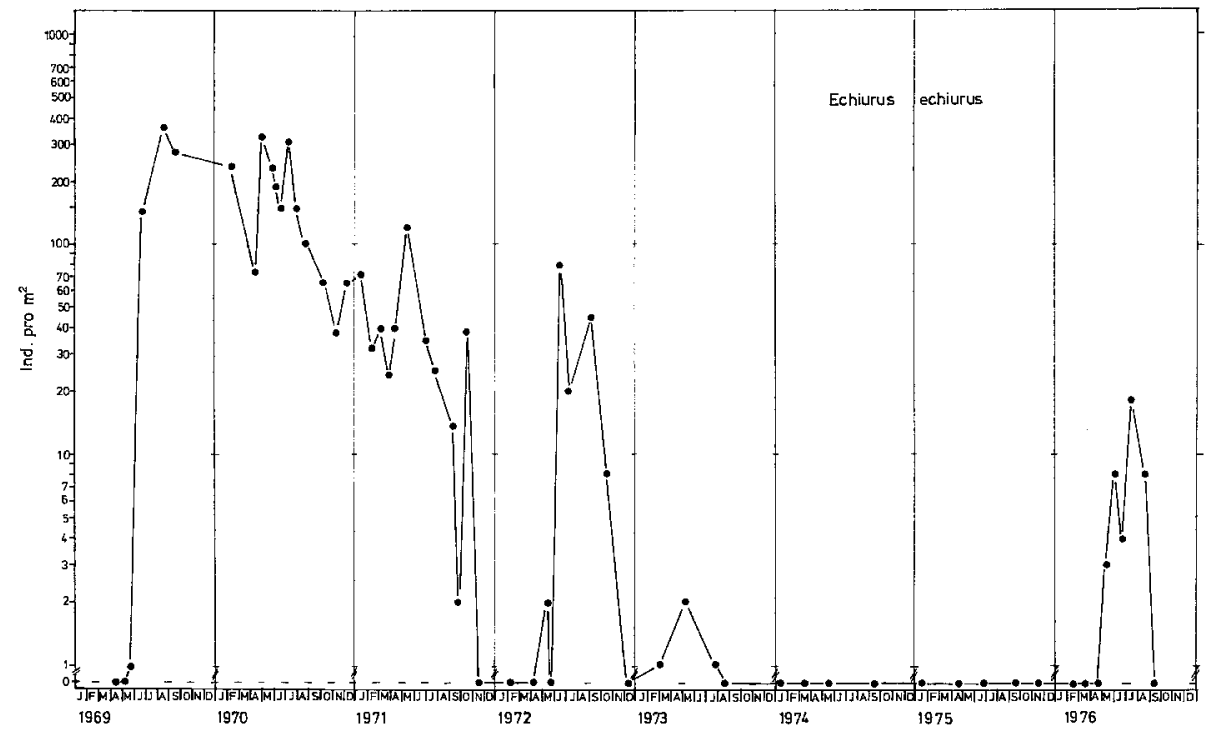

Abb. 9: Abundanz von Echiurus echiurus (Echiurida), Entwicklung von 1969 bis 1976 


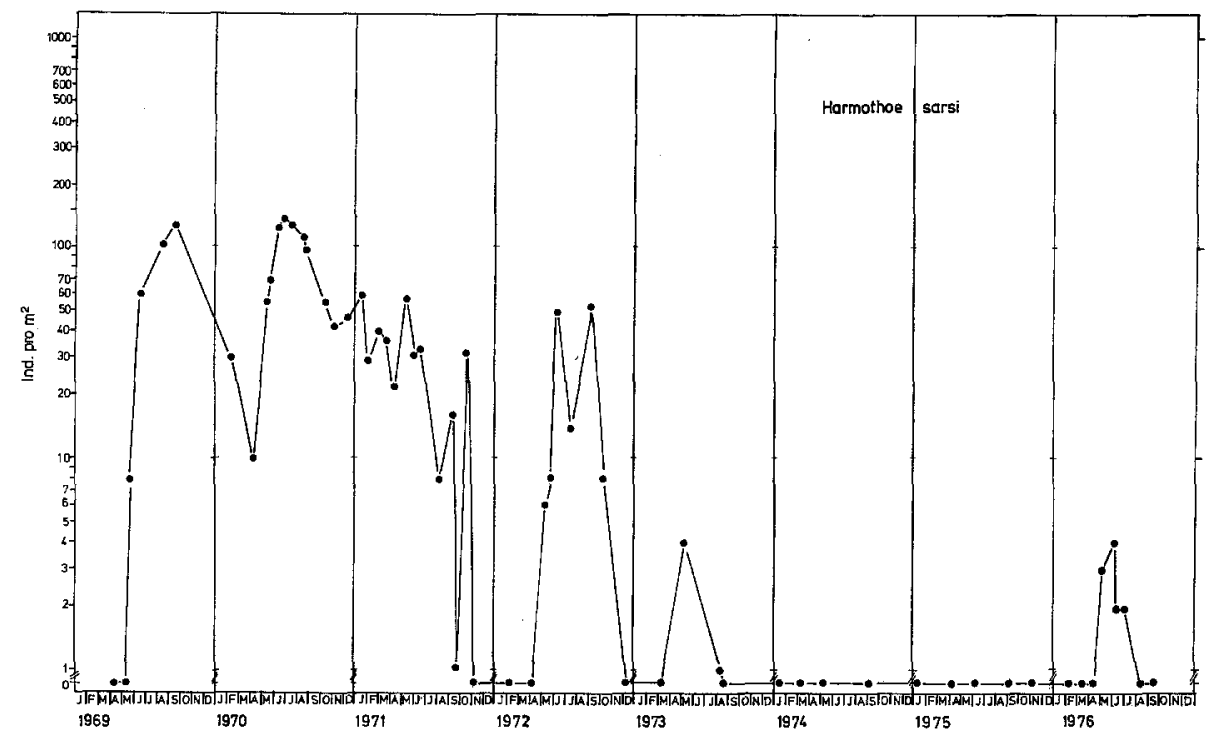

Abb. 10: Abundanz von Harmotboe sarsi (Polychaeta), Entwicklung von 1969 bis 1976

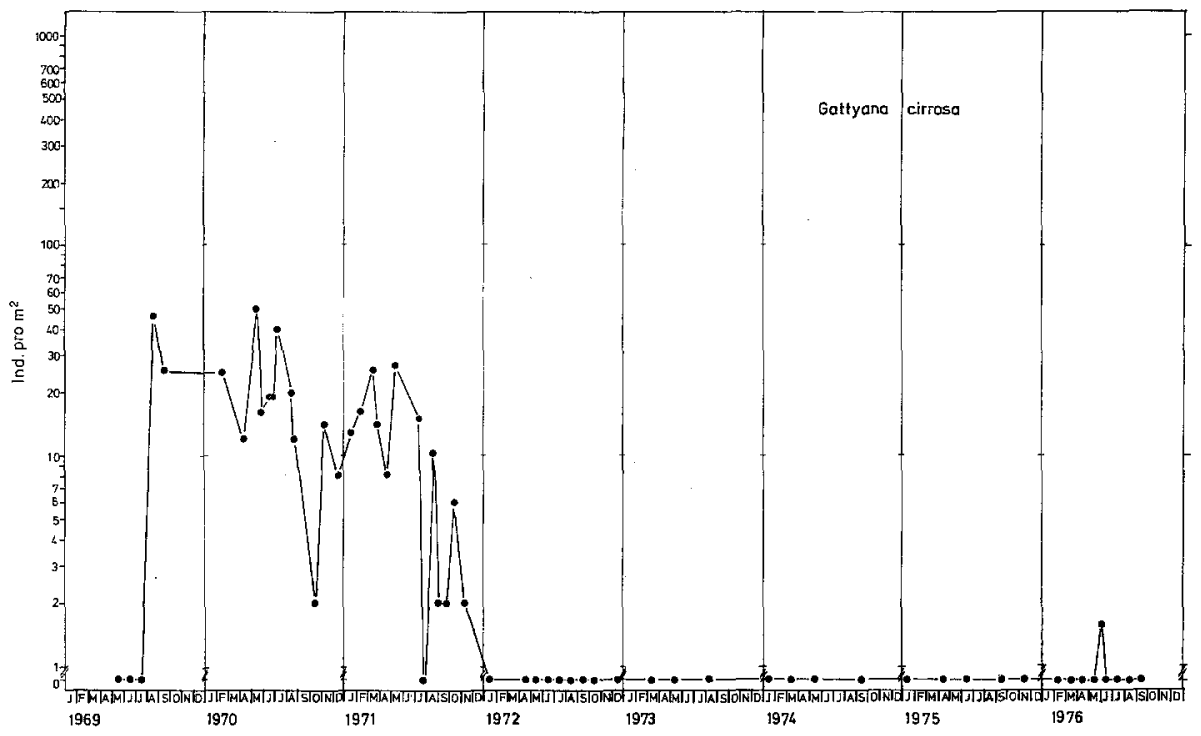

Abb. 11 : Abundanz von Gattyana cirrosa (Polychaeta), Entwicklung von 1969 bis 1976

(f) Viele seltenere Arten, die insbesondere in den Sommermonaten das Areal zu besiedeln versuchen, z. B. Ampharete acutifrons, Capitomastus minimus und Eteone longa. Die noch 1966 bzw. 1967 als charakteristisch für das Gebiet angesehene Poly chaetenart Notomastus latericeus (vgl. Dörjes, 1968; Stripp, 1969b) war im Untersuchungszeitraum 1969-1976 ohne Bedeutung (nur in zwei Proben vorhanden). 


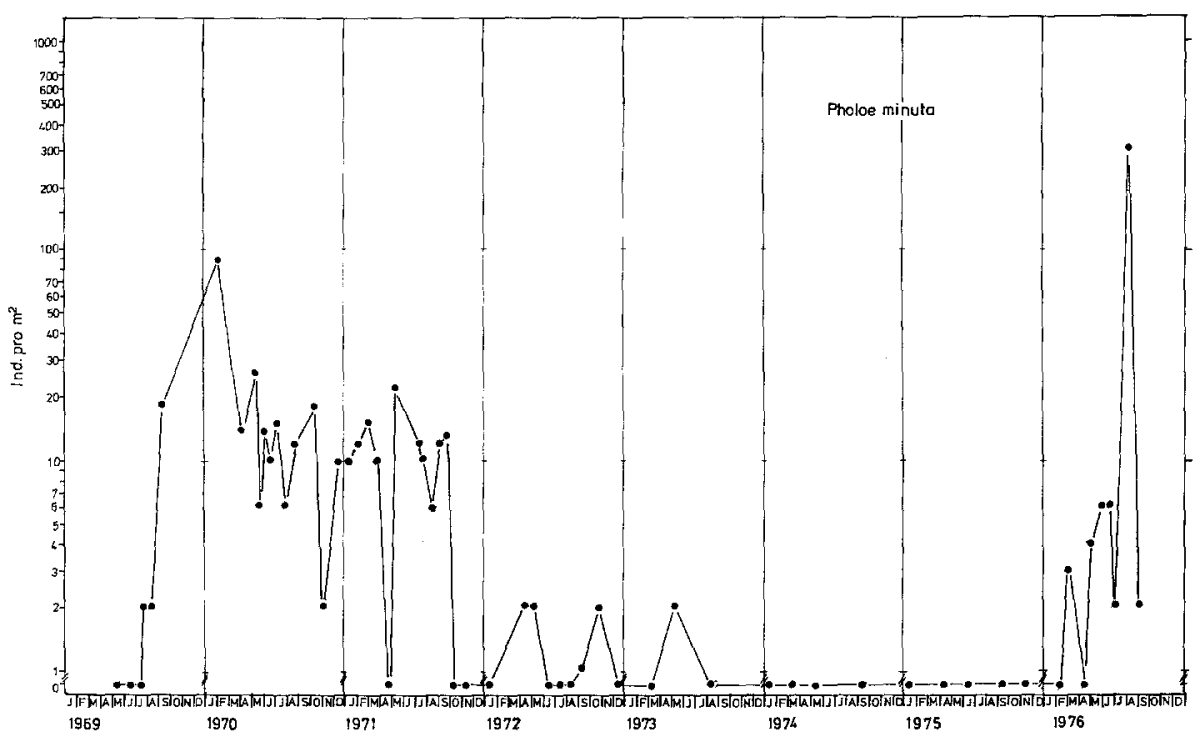

Abb. 12: Abundanz von Pholoe minuta (Polychaeta), Entwicklung von 1969 bis 1976

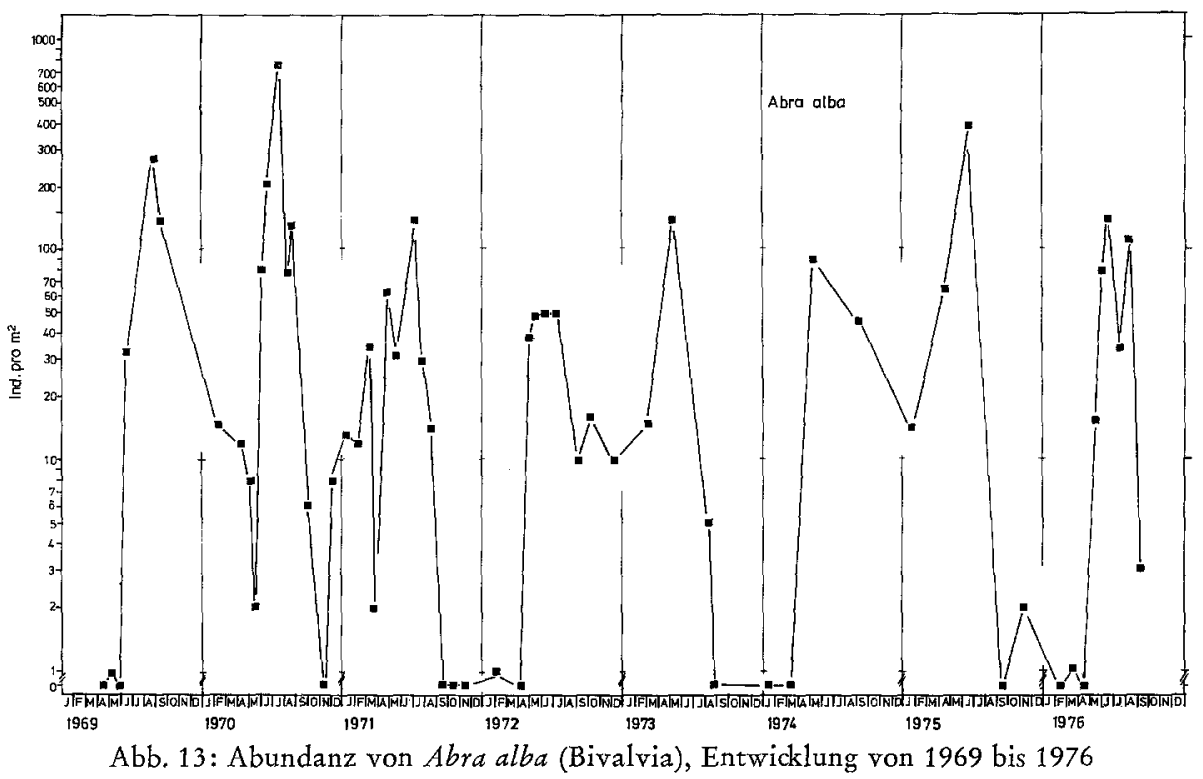

Durch stichprobenhafte Aufsammlungen in der Nachbarschaft des Untersuchungsgebietes wurde festgestellt, daß auch in der Umgebung der Station das Makrobenthos verarmt ist, insbesondere in den östlich angrenzenden Bereichen bis hin zur Verklappungsposition der Hamburger Klärschlämme. So wurden dort im Oktober 1972 nur noch die Arten Nucula nitidosa, Diastylis ratbkei, Opbiura texturata und O. albida, 
Nephtys hombergii und Paramysis kervillei mit insgesamt nur 647 Individuen pro $\mathrm{m}^{2}$ gefunden, also ein identisch verarmtes Faunenspektrum wie an der Untersuchungsstation.

\section{DISKUSSION}

Schlickbiotope zählen zu den extremen Lebensräumen des marinen Benthals. Das feine Sediment ist besonders in Küstennähe reich an organischem Detritus, ist sehr kompakt und schlecht durchlïftet; in vielen Fällen kommt es zu starken Fäulnisprozessen, in deren Gefolge $\mathrm{H}_{2} \mathrm{~S}$ freigesetzt wird. Hagmeier (1925) hat die Fauna des hier untersuchten Schlickgebietes vor den Asstuarien der Deutschen Bucht in Anlehnung an Petersen (1914) als Abra-(= Scrobicularia-)alba-Gemeinschaft bezeichnet, und auch Stripp (1969b) ist diesem Vorbild gefolgt. Die Ahnlichkeit dieser Abra-alba-Coenose zur Echinocardium-Filiformis-Gemeinschaft ist schon von Remane (1940) betont worden; Jones (1950) führt sie als mögliche Modifikation dieser Gemeinschat bei der "boreal offshore muddy sand association" auf. Insbesondere hebt sie sich durch ihre Artenarmut, nicht aber durch eigene Charakterarten von der Echinocardium-Filiformis-Gemeinschaft ab. Dörjes (1968) hat für die hier untersuchte, schlickbewohnende Assoziation den Namen Echiurus-echiurus-Coenose vorgeschlagen. Der von uns an der Untersuchungsstation und auch in ihrer Umgebung festgestellte Rückgang von Echiurus echiurus und ebenso sein Fehlen schon während der Untersuchungen von Hagmeier (1925) machen eine entsprechende Benennung fragwürdig. Die hohen Siedlungsdichten von E. echiurus während der Periode 1963-1971 (vgl. Reineck et al., 1967; Dörjes, 1968; Stripp, 1969b) sind wahrscheinlich kein normaler, andauernder Zustand, wenn auch vielleicht ein regelmäßig auftretendes Ereignis im untersuchten Schlickgebiet, etwa nach besonders kalten Wintern und Dezimierung der übrigen Fauna (Ziegelmeier, 1970).

Der Frage, ob es sich bei der hier untersuchten "klassischen" Albra-alba-Coenoese um eine eigenständige Tiergemeinschaft (nach Glémarec, 1973, auch Nucula-turgidaAbra-alba-Gemeinschaft) oder nur eine Variante z. B. der Echinocardium-FiliformisGemeinschaft handelt, soll hier nicht weiter nachgegangen werden. Hier soll nur festgehalten werden, daß für Schlickböden grundsätzlich Artenarmut und starke Fluktuationen auch zeitweise charakteristischer, dominierender Arten angenommen werden müssen.

Rückgänge im Faunenbestand des Sublitorals der Deutschen Bucht sind als besondere Ereignisse vor allem von Ziegelmeier $(1963,1964,1970)$ und auch von Rachor \& Gerlach (im Druck) beschrieben worden. Ziegelmeier hat gezeigt, daß die Fauna während extrem kalter Winter sehr stark dezimiert werden und die Wiederherstellung einer ausgewogenen Assoziation mehrere Jahre dauern kann. Rachor \& Gerlach haben insbesondere auf die Effekte von Sturmwetterlagen hingewiesen, wobei es zu direkter Schädigung der Bodentiere durch Erosion, Überschüttung und Verdriftung an ungünstige Standorte kommen kann. Außerdem ergeben sich im Gefolge von Sturmwetterlagen Umsortierungen und Veränderungen im Sediment, wodurch indirekte Einflüsse noch nicht hinreichend bekannter Art zustande kommen. So kann ein derartig verändertes Sediment z. B. attraktiv für driftende Larven werden, einen höheren oder 
niedrigeren Gehalt an organischem Detritus und somit einen veränderten Nährwert erhalten und auch günstigere oder schlechtere $\mathrm{O}_{2}$-Verhältnisse bieten. Reineck (1968) hat darauf hingewiesen, daß das hier untersuchte Schlickgebiet ebenfalls stark von Sturmwetterperioden beeinflußt wird. Dabei kann die oberste Schlicklage unter Zurücklassung von Schill erodiert und dieser dann von Sand überschüttet werden. Bei Wetterberuhigung wird darüber häufig sehr rasch das Absetzen von suspendiertem Schlick erfolgen, durch den Tiere wie Nucula nach oben flüchten. Derartige Veränderungen des Substrats werden ebenfalls direkte und indirekte Auswirkungen auf die Fauna haben.

Während unserer Untersuchungen wurden Einlagerungen von Feinsand insbesondere nach dem stürmischen Herbst 1973 im Januar und März 1974 und nach den Januarsturmfluten 1976 festgestellt. In einzelnen Bodengreifern wurden 1976 von Februar bis Ende Juni direkte Schlicksand-Überschüttungen an der Oberfläche gefunden, und der Boden war in diesen Fällen 2-3 cm tief oxydiert. Nachdem die Bodenfauna schon im Sommer 1975 nach Arten- wie Individuenzahl auf einen Tiefststand gesunken war, ist es nicht verwunderlich, daß nach dem Winter mit seinen extremen Sedimentveränderungen das absolute Besiedlungsminimum registriert wurde (nur 4 Arten und 28 Individuen pro $\mathrm{m}^{2}$ im April 1976). Die anschließende Neubesiedlung jedoch war auf Grund verbesserter Sedimenteigenschaften reichhaltig, allerdings nicht nachhaltig. Der Zusammenbruch dieser Besiedlungsphase ist offensichtlich im August erfolgt (vgl. Tab. 1).

Dieses führt uns zur Frage der kontinuierlichen Faunenverarmung im Untersuchungsgebiet. Es ist auffällig, daß verschiedene, individuenreiche Bestände insbesondere immer wieder in den Hochsommermonaten zusammengebrochen oder stark geschwunden sind: so vor allem die Arten Echiurus echiurus, Harmothoe sarsi, Gattyana cirrosa und Pboloe minuta schon im Sommer 1971 und nach der Wiederbesiedlung 1976 sowie auch die zusammen mit Scalibregma inflatum und Pectinaria koreni vorn aufgeführten Arten (Gruppe d) und vor allen Dingen Abra alba.

Diese wiederkehrenden sommerlichen Einbrüche haben insgesamt zur kontinuierlichen Verarmung der Fauna geführt. Anhaltende, durch hohe Temperaturen begünstigte Milieuverschlechterungen im Boden selbst und auch im darüberstehenden Wasser sind anzunehmen, und zwar Sauerstoffzehrung und Entwicklung von Schwefelwasserstoff. Hierfür sprechen folgende Indizien und Tatsachen aus der Biologie der Bodentiere:

(1) Das Sediment ist reich an organischem Detritus (Gadow \& Schäfer, 1973).

(2) Die oxydierte Oberflächenschicht des Sediments schrumpft in den Sommermonaten in der Regel auf weniger als $2 \mathrm{~mm}$ Schichtdicke zusammen.

(3) Vorläufige hydrographische Messungen im Sommer 1976 bestätigen das Vorhandensein einer thermohalinen Schichtung im Untersuchungsgebiet, wobei im bodennahen Wasser Sauerstoffverarmung auftritt.

(4) Im Sommer abgestorbene Abra alba hinterlassen ihre gut erhaltenen Schalen im Boden, so daß ein Wegfraß durch größere Räuber (Fische) auszuschließen ist. Nach Dries \& Theede (1974) hat Abra alba bei $20^{\circ} \mathrm{C}$ eine geringe $\mathrm{O}_{2}-\mathrm{Mangel}$ (resistenz.

(5) Die Muschel Nucula nitidosa dagegen ist offensichtlich gegenüber $\mathrm{O}_{2}$-Mangel und $\mathrm{H}_{2} \mathrm{~S}$-Bildung weniger empfindlich; sie kann bei Sommertemperaturen wenigstens 
eine Woche lang bei Oz-Mangel überleben (Rachor, 1976). Das Aufrechterhalten einer Population wird zudem dadurch ermöglicht, daß sich die Tiere erst nach der Hochsommerperiode fortpflanzen. Dennoch ist auch bei $N u c u l a$ die Abundanz rückläufig, wohl weil sie - im Gegensatz zu den anderen konstant gefundenen Arten - ganz an das Sediment gebunden ist.

(6) Die drei anderen konstanten Arten können auf Grund ihrer großen Vagilität und ihres Schwimm- und Driftvermögens ungünstigen Bodenverhältnissen ausweichen. So vermag Nephtys hombergii gut zu schwimmen und möglicherweise auch kurzfristig ungünstige $\mathrm{O}_{2}-$ Verhältnisse zu ertragen (die Art $N$. ciliata aus der Ostsee hat nach Dries \& Theede bei $15^{\circ} \mathrm{C}$ eine $\mathrm{LD}_{50}$-Zeit von fast vier Tagen). Möglicherweise ist $N$. hombergii durch mehrere Fortpflanzungsschübe im Jahr gut zum Überstehen auch ungünstiger Sommerperioden angepaßt. Ophiura texturata lebt an der Sedimentoberfläche und ist sehr vagil. Die Cumacee Diastylis ratbkei kann aktiv schwimmend ins Wasser entweichen; ihre Brut entwickelt sich während der günstigeren Jahreszeit (Herbst und Winter) im Marsupium. Alle drei hier aufgeführten Arten können dank ihrer Vagilität auch sehr schnell von günstigeren Standorten aus ein aufgelassenes Areal wiederbesiedeln.

(7) Für ungünstige Bedingungen auch im bodennahen Wasser spricht der Tatbestand, daß auch Tiere geschädigt werden, die ihren Sauerstoffbedarf aus diesem Wasser decken können, etwa durch Einstrudeln von Wasser ( $A$ bra ) oder durch kurzes Aufschwimmen (Harmothoe). Auch das Verschwinden von Echiurus echiurus und möglicherweise weiterer Arten kann dadurch erklärt werden: Normalerweise befördert E. echiurus nämlich durch seine Wühl- und besonders Pump-Tätigkeit sauerstoffreiches Wasser in den Boden hinein (Reineck et al., 1967) und kann dadurch gleichzeitig die mit ihm vergesellschafteten Tiere mit sauerstoffreichem Wasser versorgen.

Die hier beschriebene Faunenverarmung ist vergleichbar mit Erscheinungen aus belasteten Schlickbiotopen anderer Meeresgebiete. So sind aus der Ostsee Rückgänge in den tiefergelegenen Abra-alba-Coenosen auf Grund von Sauerstoffschwund schon lange bekannt (Remane, 1940). Aus der Lübecker Bucht hat Schulz (1968) Verhältnisse beschrieben, die mit den hier dargestellten gut übereinstimmen. Möglicherweise genügt als Erklärung für die abnehmenden Trends in verschiedenen Populationen und in der Artenmannigfaltigkeit die Belastung des Untersuchungsgebietes durch das zuströmende Elbwasser sowie übermäßige Planktonproduktion und -zersetzung im Mischgebiet der Konvergenzzone. Offensichtlich hat dann diese Belastung innerhalb der vergangenen Jahre noch zugenommen oder zumindest einen kritischen Wert überschritten.

In den Bereich des ausströmenden Elbwassers hinein werden nun allerdings schon seit Anfang der 60er Jahre die Klärschlämme der Hamburger biologischen Klärąnlage per Schiff eingebracht. Im Monat sind es zur Zeit etwa $28000 \mathrm{~m}^{3}$ Schlamm mit einem Feststoffgehalt von 6-10\% und einem Glühverlust von 50-54\%, d. h. etwa 1300 bis $1400 \mathrm{~m}^{3}$ oxydierbarer Feststoffe*. Es ist durchaus wahrscheinlich, daß derartige Mengen auch in einem gezeitenbeeinflußten, relativ offenen Meeresgebiet unter ungünstigen hydrographischen Bedingungen negative Auswirkungen auf die Bodenfauna mit sich

* Herrn Müller von den Hamburger Klärwerken sei für diese Angaben gedankt. 
bringen, zumindest in einem solchen Gebiet wie der hier beschriebenen Konvergenzzone, in der sich die Wasserkörper übereinanderschieben und in der die feinen Trübstoffe sedimentieren.

Außerdem ist zu berücksichtigen, daß die Menge der verklappten Klärschlämme seit 1973 um etwa ein Drittel erhöht wurde und daß der Anteil gerade der feinen Trübstoffe gewachsen ist. Für einen negativen Einfluß der Schlammverklappungen sprechen auch die hin und wieder durchgeführten Vergleichsuntersuchungen in der Nachbarschaft der Dauerstation: Bis hin zur Verklappungsstelle wird östlich unserer Dauerstation nur noch ein gleichartig verarmtes Makrobenthos gefunden, während dagegen einige $\mathrm{km}$ westlich der Station noch eine artenreichere Abra-alba-Coenose existieren kann.

Caspers (DFG-Bericht 1975) berichtet über Massenentwicklungen von Abra alba im Bereich des Schlamm-Verklappungsgebietes. Da auch diese Massenentwicklungen wieder in Zusammenbrüchen der Populationen endeten, dürften die dort zu beobachteten Erscheinungen ähnlich sein wie an der von uns untersuchten Schlickstation. Auf Grund der suspensionsartigen Konsistenz des Klärschlammes ist anzunehmen, daß Auswirkungen durch Verdriften der feinen Trübe auch - oder sogar erst recht - in wenige $\mathrm{km}$ entfernten Arealen der Konvergenzzone auftreten. Möglicherweise wird schon im Wasser ein Abbau der suspendierten, leichter oxydierbaren organischen Substanzen beginnen, wodurch starke Sauerstoffzehrungen bei hohen Temperaturen erwartet werden können. Die stetige, vermehrte Zufuhr von organischer Substanz in das Bodensediment belastet das Benthos, und in den sommerlichen Perioden starker Stagnation kommt es zum Absterben der empfindlichen Glieder der Fauna. Gelegentliche Verbesserungen des Substrats, wie nach dem Wintersturm 1976, können eine Wiederbesiedlung im Frühsommer fördern, haben aber bisher keinen wirklichen Einschnitt in den nun schon mehrere Jahre laufenden Trend der Faunenverarmung gebracht.

\section{SCHLUSSBETRACHTUNG}

Nach den hier vorgetragenen Ergebnissen ist deutlich, daß in der Hydrographie der inneren Deutschen Bucht noch viele Fragen offen sind, insbesondere über die Sauerstoff-Verhältnisse während ausgeprägter Phasen thermohaliner Schichtungen während der Sommermonate. Unsere im Detail z. T. noch vorläufigen Ergebnisse erlauben den Schluß, daß in den Bereichen der Konvergenzzone, in denen feine Trübstoffe und auch viel organische Substanz sedimentieren, in solchen Phasen eine starke $\mathrm{O}_{2}$-Zehrung und damit eine Belastung des OKosystems möglich ist. Es erscheint bedenklich, solche Gebiete für die Einbringung von Abfällen ỵorzusehen.

Selbst wenn einige der vergangenen Jahre durch wetterbedingte, hydrographische Besonderheiten aus der Regel gefallen sein sollten (etwa durch verstärkte und längerdauernde thermohaline Schichtungen im gleichen Seegebiet), bleiben diese Bedenken bestehen.

Danksagungen. Der Deutschen Forschungsgemeinschaft danke ich für die Unterstützung der durchgeführten Arbeiten (Litoralforschung - Abwässer in Küstennähe), meinem Mit- 
arbeiter, Herrn K.-H. Mantau, für die sorgfältige Mithilfe bei den Probenauswertungen und der Besatzung des Forschungsschiffes "Victor Hensen" für den Einsatz bei der Probenbeschaffung.

\section{ZITIERTE LITERATUR}

Caspers, H. (Hrsg.), 1975. Pollution in coastal waters: an interim report of the priority programme of the German Research Society. Deutsche Forschungsgemeinschaft, Bonn Bad Godesberg, $142 \mathrm{pp}$.

Dries, R.-R. \& Theede, H., 1974. Sauerstoffmangelresistenz mariner Bodenevertebraten aus der westlichen Ostsee. Mar. Biol, 25, 327-333.

Dörjes, J., 1968. Das Makrobenthos. Senckenberg. leth. 49, 272-284.

Gadow, S., 1968. Die Sedimente. Senckenberg, leth. 49, 265-269.

- \& Schäfer, A., 1973. Die Sedimente der Deutschen Bucht: Korngrößen, Tonmineralien und Schwermetalle. Senckenberg. marit. 5, 165-178.

Glémarec, M., 1973. The benthic communities of the European North Atlantic continental shelf. Oceanogr. mar. Biol. 11, 263-289.

Goedecke, E., 1968. Über die hydrographische Struktur der Deutschen Bucht im Hirblick auf die Verschmutzung in der Konvergenzzone. Helgoländer wiss. Meeresunters. 17, 108-125.

Hagmeier, A., 1925. Vorläufiger Bericht über die vorbereitenden Untersuchungen der Bodenfauna der Deutschen Bucht mit dem Petersen-Bodengreifer. Ber. dt. wiss. Kommn Meeresforsch. 1, 247-272.

Jones, N. S., 1950. Marine bottom communities. Biol. Rev. 25, 283-313.

Klein, G., Rachor, E. \& Gerlach, S. A., 1975: Dynamics and productivity of two populations of the benthic tube-dwelling amphipod Ampelisca brevicornis (Costa) in Helgoland Bight. Ophelia 14, 139-159.

Lüneburg, H., 1963. Wassermischvorgänge vor der Weser- und Elbmündung. Veröff. Inst. Meeresforsch. Bremerh. 8, 111-141.

Petersen, C. G. J., 1914. Valuation of the sea II. The animal communities of the sea-bottom and their importance for marine zoogeography. Rep. Dan. biol. Stn 21, 1-44.

Pielou, E. C., 1969. An introduction to mathematical ecology. Wiley-Interscience, New York, $286 \mathrm{pp}$.

Rachor, E., 1976. Structure, dynamics and productivity of a population of Nucula nitidosa (Bivalvia, Protobranchiata) in the German Bight. Ber. dt. wiss. Kommn Meeresforsch. 24, 296-331.

- \& Dethlefsen, V., 1974. Effects of acid-iron waste disposal in the marine environment. C.M.-ICES. E 27, 1-13.

- \& Gerlach, S. A. Changes of macrobenthos in a sublittoral sand area of the German Bight, 1967-1975. Rapp. P.-v. Réun. Cons. perm. int. Explor. Mer 172 (in press).

- \& Salzwedel, H., 1976. Studies on population dynamics and productivity of some bivalves in the German Bight. In: Proceedings of the 10th European Symposium on Marine Biology. Ed. by G. Persoone \& E. Jaspers. University Press, Wetteren, 2, 575-588.

Reineck, H.-E., 1963: Sedimentgefüge im Bereich der südlichen Nordsee. Abh. Senckenberg. naturforsch. Ges. 505, 1-138.

- 1967. Die Sedimente. Senckenberg. leth. 48, 219-275.

- 1968. Die Sturmflutlagen. Senckenberg. leth. 49, 270-272.

- Gutmann, W. F. \& Hertweck, G., 1967. Das Schlickgebiet suidlich Helgoland als Beispiel rezenter Schelfablagerungen. Senckenberg. leth. 48, 219-275.

- Dörjes, J., Gadow, S. \& Hertweck, G., 1968. Sedimentologie, Faunenzonierung und Faziesabfolge vor der Ostküste der inneren Deutschen Bucht. Senckenberg. leth. 49, 261-309.

Remane, A., 1940. Einführung in die zoologische Okologie der Nord- und Ostsee. Tierwelt Nord- u. Ostsee 1a, 1-238. 
Schulz, S., 1968. Rückgang des Benthos in der Lübecker Bucht. Mber. dt. Akad. Wiss. Berl. 10, 748-754.

Stripp, K., 1969a. Jahreszeitliche Fluktuationen von Makrofauna und Meiofauna in der Helgoländer Bucht. Veröff. Inst. Meeresforsch. Bremerh. 12, 65-94.

- 1969b. Die Assoziationen des Benthos in der Helgoländer Bucht. Veröff. Inst. Meeresforsch. Bremerh. 12, 95-142.

- 1969c. Das Verhältnis von Makrofauna und Meiofauna in den Sedimenten der Helgoländer Bucht. Veröff. Inst. Meeresforsch. Bremerh. 12, 143-148.

Ziegelmeier, E., 1963. Das Makrobenthos im Ostteil der Deutschen Bucht nach qualitativen und quantitativen Bodengreiferuntersuchungen in der Zeit von 1949-1960. Veröff. Inst. Meersforsch. Bremerh. (Sonderbd) 1, 101-114.

- 1964. Einwirkungen des kalten Winters 1962/63 auf das Makrobenthos im Ostteil der Deutschen Bucht. Helgoländer wiss. Meeresunters. 10, 276-282.

- 1970: Über Massenvorkommen verschiedener makrobenthaler Wirbelloser während der Wiederbesiedlungsphase nach Schädigungen durch "katastrophale" Umwelteinflüsse. Helgoländer wiss. Meeresunters. 21, 9-20.

Anschrift des Autors: Dr. E. Rachor Institut für Meeresforschung Bremerhaven

Am Handelshafen 12

D-2850 Bremerhaven

Bundesrepublik Deutschland 